\title{
LA VOLATILIDAD DE LOS MERCADOS FINANCIEROS GLOBALIZADOS: IMPACTO EN LA BOLSA DE VALORES DE LIMA - PERU
}

\author{
THE VOLATILITY OF GLOBALIZED FINANCIAL MARKETS: IMPACT ON THE \\ STOCK EXCHANGE OF LIMA - PERU
}

Pedro Pablo Chambi Condori

Doctor en Administración y Dirección de Empresas por la Universidad Inca Garcilaso de la Vega - Lima, Perú

Email: ppchc2007@yahoo.es (Autor Corresponsal)

[Recibido: 14/08/2016 Aceptado: 18/11/2016]

\begin{abstract}
RESUMEN:
El estudio de la volatilidad de la rentabilidad de los mercados financieros internacionales y su incidencia en la rentabilidad de la Bolsa de Valores de Lima es muy importante para los agentes que toman parte en los mercados: Los inversionistas del mercado de acciones. El estudio de la verificación de la estacionariedad de la rentabilidad de los diferentes mercados bursátiles, el estudio de la volatilidad condicionada es tan igual de importante para los inversionistas. Que según el comportamiento que se visualizan a través de los indicadores, los inversionistas tienen una herramienta para cualificar sus decisiones en la conformación de sus carteras de inversión en activos financieros riesgosos. El objetivo de la investigación consiste en determinar el tipo de volatilidad de la rentabilidad de la BVL, y las incidencias que recibe de los otros mercados financieros globales. Para lo que se ha recogido la data diaria de la cotización en INDICES para luego a partir de las series, obtener la rentabilidad diaria, y mediante el procesamiento estadístico de los datos recogidos, se desarrollan las pruebas que se han utilizado para determinar el proceso autoregresivo y la heterocedasticidad condicionada mediante el modelado ARCH(1) y GARCH(1,1) y la prueba de raíz unitaria de Dickey - Fuller para calificar la estacionariedad de la serie y por otro lado en modo gráfico y mediante la matriz de correlación mostrar la incidencias que existen entre diversos mercados financieros globales.
\end{abstract}

PALABRAS CLAVE:

Volatilidad, heterocedasticidad, rentabilidad, garch.

\begin{abstract}
ABSTRAC:
The study of the profitability's volatility of the of international financial markets and its impact on the profitability of the Lima Stock Exchange is very important for the agents that take part in the markets: Stock market investors. The study of stationarity verification of the different stock markets' profitability and the study of conditioned volatility is just as important for investors. According to the behavior showed through the indicators, the investors have a tool to qualify their decisions in the conformation of their portfolios of investment in risky financial assets. The objective of the investigation is to determine the type of profitability's volatility of the LSE and the impacts it receives from the other global financial markets. The daily data has been collected by INDEX and later from the series, to obtain the daily profitability, and through the statistical processing of the collected data, the tests that have been used to determine the autoregressive model and conditioned by modeling heteroscedasticity ARCH (1) and GARCH $(1,1)$ and the Dickey - Fuller test for unit root to qualify the stationarity of the series and on the other graphical and using the correlation matrix showing the incidences among various global financial markets.
\end{abstract}

\section{KEYWORDS:}

Volatility, heteroscedasticity, profitability, garch.

Como Citar: Chambi, P. (2017). La volatilidad de los mercados financieros globalizados: Impacto en la Bolsa de Valores Lima - Perú. Quipukamayoc, 25(47), 103 - 111. doi: http://dx.doi.org/10.15381/quipu.v25i47.13808 


\section{INTRODUCCIÓN}

La volatilidad es una característica fundamental de los mercados financieros globales, cuya medida y previsión es de vital importancia para los que en ellos operan, de manera que su ausencia puede llegar a vaciar de contenido la operatividad en dichos mercados.

La volatilidad es una medida de la velocidad del mercado, qué tan rápido se ajustan los precios de los activos financieros ante determinados hechos. Los mercados que se mueven despacio son mercados de baja volatilidad, los mercados que se mueven de prisa son mercados de alta volatilidad. Se puede intuir que algunos mercados son más volátiles que otros. Si se puede cuantificar la volatilidad futura de los mercados de valores y se logra introducir en un modelo teórico de valoración, cualquier valor obtenido será más fiable que si simplemente se hubiera ignorado la volatilidad.

Debido a los movimientos incontrolables que se presentan en las economías, es necesario fomentar la búsqueda de métodos de medición, cálculo y control de todos los factores que diariamente afectan el desenvolvimiento de los mercados financieros, afectados primordialmente por una gran volatilidad en cuanto al precio y el volumen de las transacciones que continuamente se ven perturbadas por los movimientos bruscos de la oferta y demanda de los instrumentos que operan los agentes económicos que intervienen en estas operaciones, quienes son los que deciden a través de sus operaciones, que esta variabilidad sea más difícil de definirse y calcularse.

Estudios realizados indican que la volatilidad puede ser capturada por modelos de varianza condicionada como los modelos de la familia arch, quienes son capaces de poder predecir la varianza futura de un activo. En el presente trabajo se modela la volatilidad de los mercados globales, el comportamiento de la volatilidad que tiene el mercado de valores del Perú y la relación que tiene éste con los otros mercados del mundo, para efectos de la experiencia en el presente trabajo se utilizan los índices de los mercados más representativos del mundo, de Estados Unidos, Europa, Asia y América Latina, periodo de 1996 a 2012, en razón de un periodo especial de alta volatilidad, que incluye el periodo de la crisis financiera internacional.

Para capturar las características de la volatilidad, Engle(1982)propone modelar la volatilidad condicional por medio de un proceso de heterocedasticidad condicional autoregresiva (ARCH), en el cual la media del proceso es cero y la varianza condicional depende de los errores aleatorios pasados. El modelo viene definido por la siguiente expresión: $\sigma_{t}^{2}=\partial_{0}+\partial_{1} \varepsilon_{t-1}^{2}$

"El ARCH que quiere heterocedasticidad condicional autoregresiva, permite el modelaje condicional de la varianza, en lugar de la traidcional estimación incondicional que se hace bajo el supuesto de homecedasticidad, donde la varianza del error es una constante $\sigma^{2}$. La hipótesis de expectativas racionales postula que los agentes no desdeñan ni malgastan información útil. En muchos casos, como en las negociaciones salariales o la inversión en acciones, las decisiones que se toman dependen de la varianza o incertidumbre predicha para el periodo futuro relevante y no para el futuro en general, (...). En situaciones como éstas, que involucran o necesitan la predicción de la incertidumbre futura será beneficioso el modelaje condicional de la varianza". (Montenegro, 2010, p.198).

"La identificación de efectos ARCH se efectúa normalmente después de ajustar un modelo ARMA a la serie para eliminar la dependencia en la media. Si existen efectos ARCH, los residuos del modelo ARIMA estarán incorrelados pero no serán independientes y este efecto será visible en la función de autocorrelación de los residuos al cuadrado. (...). Para detectar estructura en los cuadrados se puede acudir a la prueba de LcLeod y Li(1983)". (Peña, 2010, p.456).

Montenegro(2010), describe, "en un modelo ARCH, simultáneamente se presentan tres distintas especificaciones: primero, media condicional; segundo, la varianza condicional; y finalmente, una para la distribución condicional del error”.(p.126).

El Modelo $\operatorname{ARCH}(p, q)$ generalizado, el llamado modelo GARCH(p,q), disponible para las series temporales autoregresivos y con varianza heterocedástica (Enders, 2015).

El modelado de la rentabilidad se realiza haciendo, Las variaciones en el precio de cierre del índice son la causa de rentabilidad que este genera. A partir de los precios diarios (cierre de los índices) se calcula la rentabilidad diaria como una variable aleatoria a la volatilidad de las rentabilidades.

Se define la Rentabilidad Precio como el cociente de la diferencia de precios entre el período $t$ y el período $t-1$, y el precio en el período $\mathrm{t}-1$ y se tiene el modelo: 
$Y_{t}=\left(P_{t}-P_{t-1}\right) / P_{t-1}$

Se utiliza la rentabilidad logarítmica, que para valores pequeños de la Rentabilidad Precio resulta ser una buena aproximación de la rentabilidad real, y permite la suma de las rentabilidades.

$$
Y_{t}=\ln \left(P_{t}-P_{t-1}\right)
$$

Se asume el supuesto que el logaritmo del precio verifica la ecuación:

$\ln P_{t}=r_{o}+\ln P_{t-1}+a t$

Donde $\mathrm{r}_{\mathrm{o}}$ es una constante $\mathrm{y}$ at es una variable aleatoria normal con media cero y varianza $\sigma^{2}$, y que se distribuye idéntica e independientemente a lo largo del tiempo.

Entonces se puede escribir la rentabilidad logarítmica como:

$Y_{t}=r_{o}+a t$

"Si se aplica la hipótesis de que las variables aleatorias son independientes, podemos suponer que la rentabilidad de hoy no influye en la rentabilidad de mañana” (Perez, 2010, p.405).

Por otro lado es preciso señalar:

"un aspecto bastante importante y que el modelo ARCH es capaz de capturar, es el conocido como movimientos de volatilidad agrupados. Los movimientos de volatilidad agrupados se observan frecuentemente en las series de retornos de las acciones cotizadas en bolsa de valores. Estos movimientos se deben a que cambios en los precios de las acciones tienden a ser seguidos de grandes cambios, de cualquier signo, y pequeños cambios son seguidos por otros pequeños cambios. Este movimiento en los retornos, a su vez, genera similar comportamiento en la volatilidad del instrumento financiero".(Court, 2011, p.454).

El modelo GARCH $(1,1)$ fue propuesto para casos de series financieras que tienen exceso de curtosis y el agrupamiento de la volatilidad. El modelo proporciona una manera adecuada de pronosticar las varianzas y covarianzas de los retornos de los activos. Engle(2002), el modelo GARCH tiene aplicación en el tratamiento de administración de riesgos, en la administración de portafolio de inversiones, en la asignación de activos, en las opciones de precio, en las tasas de cambio, en la tasa de interés, en mercados accionarios.

$$
\sigma_{t}^{2}=\partial_{0}+\partial_{1} \varepsilon_{t-1}^{2}+\theta_{1} \sigma_{t-1}^{2}
$$

en donde

$$
\partial_{0}>0, \partial_{1} \geq 0, \theta_{1} \geq 0 \text { y } 0 \leq\left(\partial_{1}+\theta_{1}\right)<1 \text {. }
$$

Engle(2003), de acuerdo al modelo $\operatorname{GARCH}(1,1)$, se tiene que la varianza condicional heterocedástica del proceso en el periodo $t$ depende de la perturbación al cuadrado y de la varianza condicional observados en el periodo t-1. El coeficiente captura el efecto ARCH, es decir, mide la amplitud con la cual los efectos de volatilidades pasadas alimentan volatilidades presentes, el coeficiente captura el efecto GARCH y $\left(\partial_{1}+\theta_{1}\right)$ mide en el tiempo la persistencia de la volatilidad condicional, si la suma de estos coeficientes es cercana a uno, se dice que hay una elevada persistencia, de lo contrario es baja.

Hernández (2009), enfatiza, "La varianza condicional depende de tres términos: la media $\partial \_o$, información sobre la volatilidad del periodo anterior medido por el rezago de los residuales al cuadrado (término ARCH) y la varianza pronosticada del último periodo (GARCH).”(p.28).

La autocorrelación en las series temporales, Perez (2006) "para analizar la autocorrelación de un modelo suele comenzarse por el análisis gráfico de los residuos, siendo esencial la gráfica de los residuos respecto al índice tiempo, que debe de presentar una estructura aleatoria libre de tendencia. También se pueden graficar los residuos respecto de su retardo y si la mayoría de los puntos caen en el primer y tercer cuadrante hay indicios de una autocorrelación positiva, siendo la autocorrelación negativa cuando los puntos caen en el segundo y cuarto cuadrante. Aparte del análisis gráfico es necesario realizar contrastes formales de autocorrelación, por ejemplo el contraste de Durbin Watson(p.406-407).

En relación a la estacionariedad, Larios, Alvarez y Quineche(2014), afirman que:

"La estacionariedad de un proceso estocástico puede ser catalogada según la constancia de sus momentos (parámetros estadísticos). Por ello, existen dos tipos de estacionariedad(fuerte y débil). La estacionariedad fuerte la cumplen la mayoría de procesos estocásticos(series de tiempo) y se cumple cuando los valores de su media, su varianza y su autocovarianza permanecen iguales o constantes sin importar el momento en el cual se midan, es decir, son invariantes respecto del tiempo. (...). Que las series de tiempo sean estacionarias, es muy importante, debido a que la serie no fuera estacionaria, sólo podríamos estudiar su comportamiento durante el periodo en consideración, por lo que no sería posible generalizar para otros periodos y el propó- 
sito de pronosticar no se podría cumplir."(p.400-401).

Para el estudio se recoge la data histórica a través de los repositorios de los índices de los diversos países: el Índice General de la Bolsa de Valores de Lima (IGBVL), IBEX 35 de Madrid España. S\&P500 de Estados Unidos, Bovespa de Brasil y SSCE de Shangai China, comprendido en el periodo entre 1996 a 2012, periodo que incluye la crisis financiera internacional. A través de los índices se tienen los datos de cotización diaria al cierre de

\section{BVL}

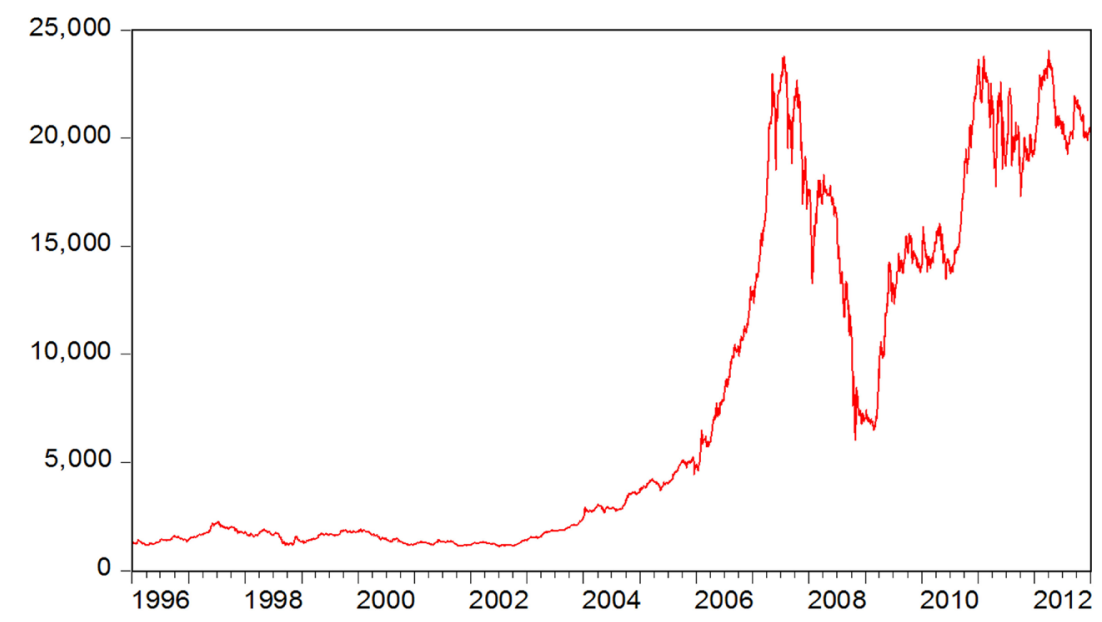

Figura 1. Comportamiento del IGVBL del periodo 1996-2012.

Fuente: Bolsa de Valores de Lima, elaboración propia.

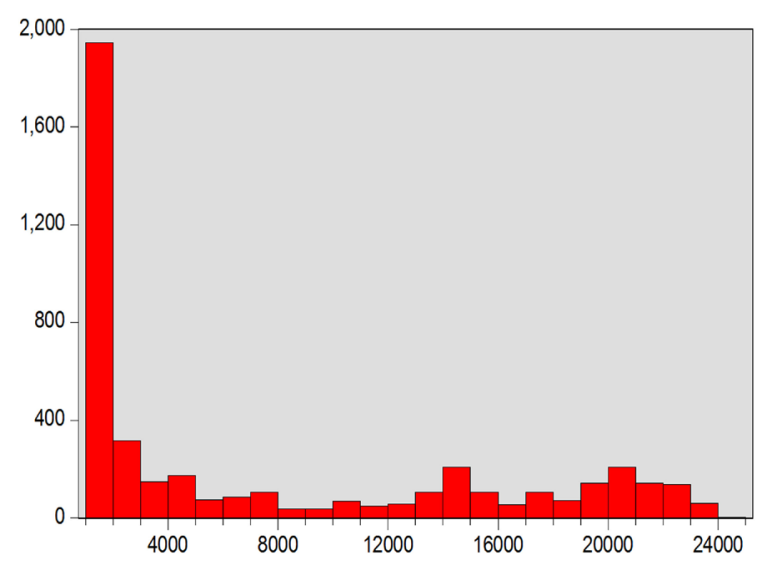

Series: BVL

Sample 1/01/1996 12/31/2012

Observations 4436

Mean $\quad 7699.061$

Median $\quad 2884.675$

Maximum 24051.62

Minimum $\quad 1109.890$

Std. Dev. $\quad 7661.290$

Skewness $\quad 0.792178$

Kurtosis $\quad 1.998693$

Jarque-Bera $\quad 649.2819$

Probability $\quad 0.000000$

Figura 2. Estadísticas principales de la serie de la BVL Fuente: Bolsa de Valores de Lima, elaboración propia

La Volatilidad de la Bolsa de Valores de Lima.

El comportamiento histórico de la Bolsa de Valores de Lima, en el periodo 1996 a 2012 se ilustra en el gráfico que se expone en líneas abajo, teniendo en cuenta la data que se obtiene del IGBVL, al precio de cierre diario.

Los principales momentos obtenidos de la evolución diaria del IGVBL a precios de cierre pueden ser vistos en la Figura 2.

\section{Análisis del comportamiento de la rentabilidad diaria de la BVL.}

Para el cálculo de la rentabilidad diaria, se ha utilizado los precios diarios de cierre del IGBVL de los días en los que hubo mercado. Los rendimientos se definen como la variación porcentual del logaritmo natural del precio de cierre del índice para dos días consecutivos de mercado. Así, el rendimiento diario se calcula de la siguiente forma:

$\mathrm{y}_{\mathrm{t}}=100 \cdot\left(\ln \mathrm{P}_{\mathrm{t}}-\ln \mathrm{P}_{\mathrm{t}-1}\right)$, expresado en términos porcentuales.

Donde: $\mathrm{y}_{\mathrm{t}}$ es el retorno para el día $\mathrm{t}$

$\mathrm{P}_{\mathrm{t}}$ representa el valor del índice al cierre del día $t$.

En lo referente a la estacionariedad de la serie, de la visualización de la serie en la Figura 3, se puede afirmar a priori que la serie es estacionaria en la media pero no así en la varianza, pues esta no parece constante.

\section{Rentabilidad diaria de la BVL}

Iniciamos el análisis de la rentabilidad, viendo primero su histograma y sus estadísticas principales expuestas en las figuras 2,3 y 4 .. 
En estas gráficas se muestran los siguientes estadígrafos descriptivos: media, desviación estándar, skewness (medida de asimetría), kurtosis, (analiza el grado de concentración que presentan los valores alrededor de la zona central de la distribución) y el estadístico Jarque-Bera con su respectiva probabilidad de que este exceda al valor observado bajo la hipótesis nula. Un pequeño valor permitiría rechazar la hipótesis nula de que la serie estocástica analizada proviene de una distribución de densidad del tipo normal.

De acuerdo a los indicadores estadísticos, la rentabilidad media en el horizonte del estudio hasta el 2012/12/31, es del orden de $0,0630 \%$ en términos porcentuales, tiene una rentabilidad máxima de $12,82 \%$ y ha registrado a lo largo del tiempo una rentabilidad mínima de -13,29\%.

$1^{\circ}$. La Curtosis es de 14.10 muy superior a la que corresponde a una distribución normal estándar de 3 .

2o. El valor de la Asimetría (Skewness) es de 0,47 levemente superior al correspondiente valor de la distribución normal estándar de 0 , indicando una cola derecha mayor a lo normal.

$3^{\circ}$. El estadístico Jarque-Bera alcanza un valor de 22492.53, muy alto, lo que nos lleva a rechazar la hipótesis de normalidad.

\section{Test de Dickey - Fuller}

El test de Dickey - Fuller aplicado a la serie del INDICE de la BVL tiene la salida que se expone en el gráfico del cuadro siguiente, para desarrollar la prueba de estacionariedad de la serie en análisis.

Se observa que el índice de bolsa de valores de lima no es estacionaria a los tres niveles críticos, puesto que el va-

BVL

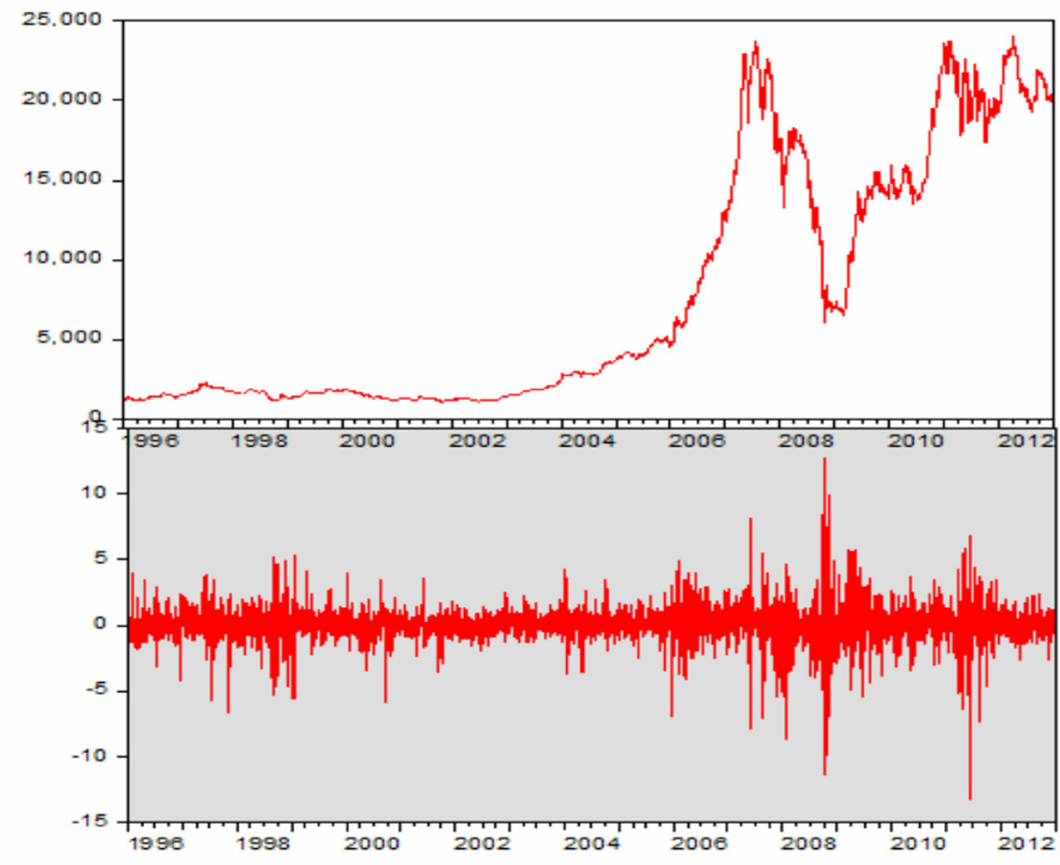

Figura 3. Volatilidad de la BVL

Fuente: Bolsa de Valores de Lima, elaboración propia.

lor ADF, -1.881012 en valor absoluto es menor al valor absoluto de los tres niveles de Mackinnon, de donde se infiere que la serie tiene raíz unitaria y por tanto no es estacionaria.

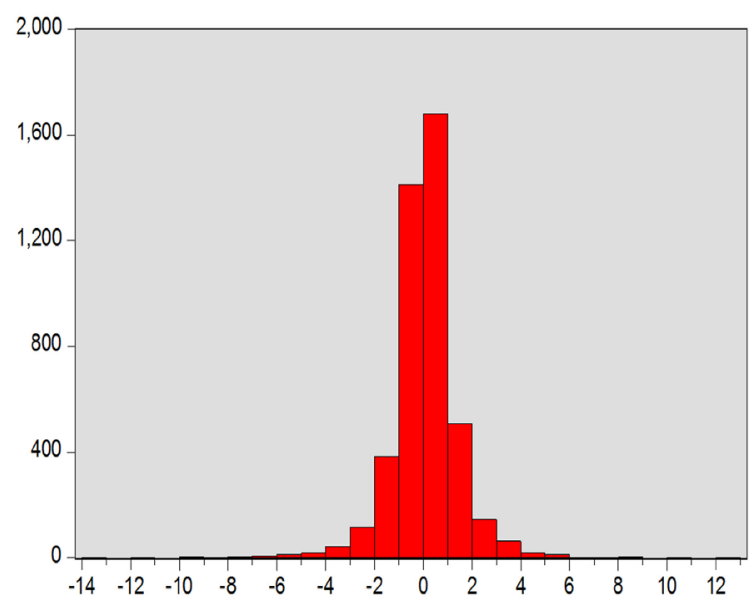

Figura 4. Estadísticas de la rentabilidad de la BVL

Fuente: Bolsa de Valores de Lima, elaboración propia.

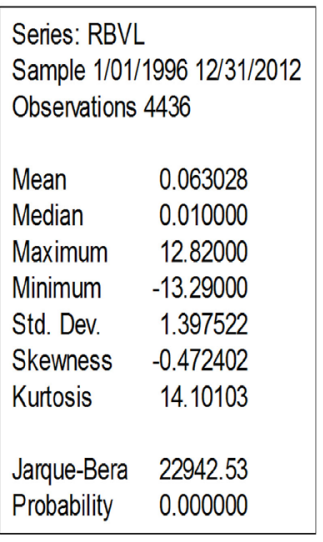

Luego realizamos la prueba de Dickey Fuller a la serie de la rentabilidad de la BVL. 
Tabla 1.

Prueba de Dickey - Fuller:Serie BVL

Fuente: Elaboración propia

\begin{tabular}{llll} 
& & t-Statistic & Prob. $^{*}$ \\
Augmented Dickey-Fuller Test Equation & $-1,881012$ & 0,6642 \\
\hline Test critical values: & $1 \%$ level & $-3,960108$ & \\
\hline $5 \%$ level & $-3,410818$ & \\
\hline $10 \%$ level & $-3,127206$ & \\
\hline
\end{tabular}

${ }^{\star}$ MacKinnon (1996) one-sided p-values.

Tabla 2.

Prueba de Dickey Fuller: Serie Rentabilidad de la BVL.

Fuente: Elaboración propia

\begin{tabular}{llll} 
& & t-Statistic & Prob. $^{*}$ \\
Augmented Dickey-Fuller test statistic & $-28,71734$ & 0 \\
\hline Test critical values: & $1 \%$ level & $-3,431656$ & \\
\hline & $5 \%$ level & $-2,862002$ & \\
\hline & $10 \%$ level & $-2,567059$ \\
\hline
\end{tabular}

*MacKinnon (1996) one-sided p-values.

Augmented Dickey-Fuller Test Equation

Dependent Variable: D(RBVL)

Method: Least Squares

Date: 06/21/17 Time: 11:51

Sample (adjusted): 54394

Included observations: 4390 after adjustments

\begin{tabular}{lllll} 
Variable & Coefficient & Std. Error & t-Statistic & Prob. \\
RBVL(-1) & $-0,754463$ & 0,026272 & $-28,71734$ & 0 \\
\hline $\mathrm{D}(\mathrm{RBVL}(-1))$ & $-0,072527$ & 0,023524 & $-3,083046$ & 0,0021 \\
\hline $\mathrm{D}(\mathrm{RBVL}(-2))$ & $-0,12083$ & 0,019485 & $-6,201028$ & 0 \\
\hline $\mathrm{D}(\mathrm{RBVL}(-3))$ & $-0,058203$ & 0,015069 & $-3,862371$ & 0,0001 \\
\hline $\mathrm{C}$ & 0,047865 & 0,020791 & 2,302259 & 0,0214 \\
\hline R-squared & 0,421954 & Mean dependent var & 0,000173 \\
\hline Adjusted R-squared & 0,421427 & S.D. dependent var & 1,805269 \\
\hline S.E. of regression & 1,37316 & Akaike info criterion & 3,473244 \\
\hline Sum squared resid & 8268,212 & Schwarz criterion & 3,480519 \\
\hline Log likelihood & -7618.771 & Hannan-Quinn criter. & 3,475811 \\
\hline F-statistic & 800.2255 & Durbin-Watson stat & 2,000835 \\
\hline Prob(F-statistic) & 0 & &
\end{tabular}

A partir de la Tabla 2, observando los indicadores del test de Dickey Fuller aplicado a la serie estadística, teniendo en cuenta que el valor absoluto ADF es mayo al valor absoluto de los tres niveles de Mackinnon, por tanto la serie de la rentabilidad de la BVL es estacionaria.

En la Tabla 2 se muestra los resultados alcanzados luego realizar la prueba de Dickey Fuller a la serie de la rentabilidad de la BVL.

A partir de la Tabla 2, observando los indicadores del test de Dickey Fuller aplicado a la serie estadística, teniendo en cuenta que el valor absoluto ADF es mayo al valor absoluto de los tres niveles de Mackinnon, por tanto la serie de la rentabilidad de la BVL es estacionaria.

\section{Verificación de la presencia del pro- ceso $\operatorname{GARCH}(1,1)$}

Luego finalmente para obtener el modelo que describe la volatilidad condicionada de la rentabilidad de la Bolsa de Valores de Lima en el periodo analizado, con el soporte del software Eviews, utilizamos el modelo GARCH $(1,1)$.

El modelo GARCH $(1,1)$ obtenido es el siguiente:

$$
\begin{gathered}
\sigma_{t}^{2}=\partial_{0}+\partial_{1} \varepsilon_{t-1}^{2}+\theta_{1} \sigma_{t-1}^{2} \\
\sigma_{t}^{2}=0,084812+0,738096 \varepsilon_{t-1}^{2} \\
+0,22474 \sigma_{t-1}^{2}
\end{gathered}
$$

En donde se puede apreciar que la suma de los coeficientes $\left(\partial_{1}+\theta_{1}\right)$ es 1,046 cercano a uno, por tanto, según Pérez(2010) podemos afirmar que el efecto de la volatilidad en la Bolsa de Valores de Lima en el periodo analizado es persistente, y además se infiere, que el comportamiento de la rentabi- 
lidad de la Bolsa de Valores de Lima responde al tipo de volatilidad condicionada y estacionaria.

La incidencia de los Mercados Globales en la Bolsa de Valores de Lima, Perú

La volatilidad del mercado global incide en la volatilidad de la rentabilidad de la Bolsa de Valores de Lima.

Para describir, de cómo incide, el comportamiento de los otros mercados al mercado de valores de Lima, se hace mediante el tratamiento de un modelo múltiple. a continuación se muestra la volatilidad a precios de cierre de los índices de las siguientes bolsas: BVLBOVESPA, IBEX-35, SHANGHAI y S\&P500 en un solo gráfico.

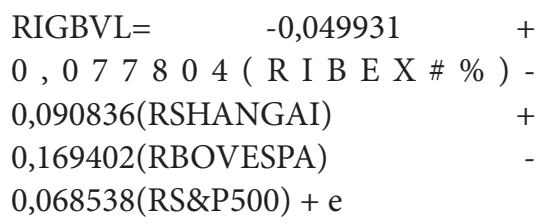

$$
\begin{aligned}
\sigma_{t}^{2} & =0,084812+0,738096 \varepsilon_{t-1}^{2} \\
& +0,22474 \sigma_{t-1}^{2}
\end{aligned}
$$

Con lo que queda demostrado que efectivamente existe relación entre la rentabilidad de la Bolsa de Valores de Lima con los otros mercados financieros globales.

\section{CONCLUSIONES}

De los resultados expuestos en líneas arriba, se confirma el proceso autoregresivo y de la heterocedasticidad condicionada presente en la serie de la rentabilidad de la Bolsa de Valores de Lima, y por otro lado, por los resultados del test de Dickey Fuller a los tres niveles críticos de Mackinnon se verifica la estacionariedad de la serie. La incidencia de los mercados financieros globales sobre la rentabilidad

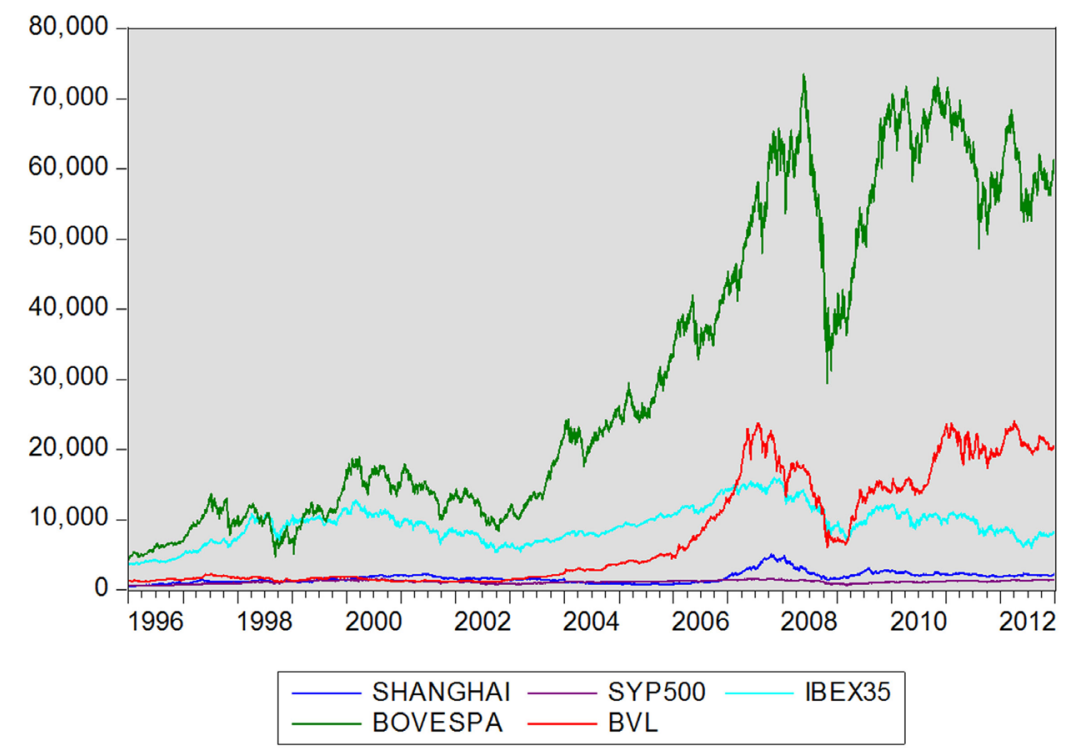

Figura 5. Volatilidad conjunta de la Bolsas en estudio Fuente: elaboración propia

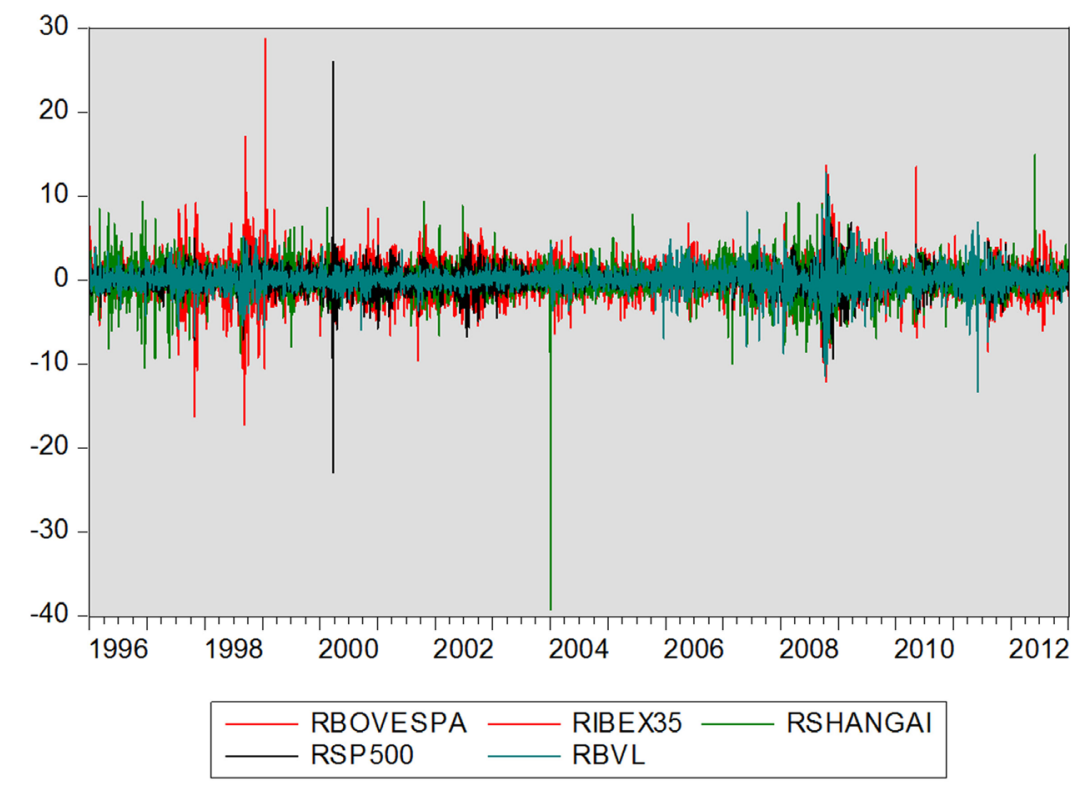

Figura 6. Comparación de la volatilidad de rentabilidad de las 5 bolsas Fuente: elaboración propia

de la Bolsa de Valores de Lima se aprecia confirmada con los resultados expuestos en las tablas 4 y 5 . De la suma total de los coeficientes del modelo $\operatorname{Garch}(1,1)$ obtenido para la serie de la rentabilidad de la Bolsa de Valores de Lima que hace 1.00 , se confirma la persistencia de la volatilidad en el tiempo.

De los resultados presentados en la 
Tabla 4.

La Bolsa de Valores de Lima en función de las demás bolsas

Fuente: Elaboración propia

Dependent Variable: RBVL

Method: ML ARCH - Normal distribution (BFGS / Marquardt steps)

\begin{tabular}{|c|c|c|c|c|}
\hline \multicolumn{5}{|c|}{ Date: $06 / 20 / 17$ Time: $15: 40$} \\
\hline \multicolumn{5}{|c|}{ Sample (adjusted): 151} \\
\hline \multicolumn{5}{|c|}{ Included observations: 51 after adjustments } \\
\hline \multicolumn{5}{|c|}{ Convergence achieved after 23 iterations } \\
\hline \multicolumn{5}{|c|}{ Coefficient covariance computed using outer product of gradients } \\
\hline \multicolumn{5}{|c|}{ Presample variance: backcast (parameter $=0,7$ ) } \\
\hline \multicolumn{5}{|c|}{$\mathrm{GARCH}=\mathrm{C}(6)+\mathrm{C}(7)^{\star} \mathrm{RESID}(-1)^{\wedge} 2+\mathrm{C}(8)^{\star} \mathrm{GARCH}(-1)$} \\
\hline Variable & Coefficient & Std. Error & z-Statistic & Prob. \\
\hline RIBEX35 & 0,077804 & 0,119389 & 0,651689 & 0,5146 \\
\hline RSHANGAI & $-0,090836$ & 0,097045 & $-0,936012$ & 0,3493 \\
\hline RBOVESPA & 0,169402 & 0,083907 & 2,018933 & 0,0435 \\
\hline RS\&P500 & $-0,068538$ & 0,121959 & $-0,561979$ & 0,5741 \\
\hline \multirow[t]{2}{*}{$\mathrm{C}$} & $-0,049931$ & 0,15386 & $-0,324522$ & 0,7455 \\
\hline & \multicolumn{4}{|c|}{ Variance Equation } \\
\hline $\mathrm{C}$ & 0,246199 & 1,021111 & 0,241109 & 0,8095 \\
\hline $\operatorname{RESID}(-1)^{\wedge} 2$ & $-0,033247$ & 0,143882 & $-0,231073$ & 0,8173 \\
\hline GARCH(-1) & 0,607768 & 1,68028 & 0,361706 & 0,7176 \\
\hline R-squared & 0,267784 & \multicolumn{2}{|c|}{ Mean dependent var } & $-0,061373$ \\
\hline Adjusted R-squared & 0,204113 & \multicolumn{2}{|c|}{ S.D. dependent var } & 0,890865 \\
\hline S.E. of regression & 0,794763 & \multicolumn{2}{|c|}{ Akaike info criterion } & 2,579817 \\
\hline Sum squared resid & 29,05579 & \multicolumn{2}{|c|}{ Schwarz criterion } & 2,882849 \\
\hline Log likelihood & $-57,78534$ & \multicolumn{2}{|c|}{ Hannan-Quinn criter. } & 2,695614 \\
\hline Durbin-Watson stat & 2,001075 & & & \\
\hline
\end{tabular}

tabla 4, observando los coeficientes del modelo multilineal, se aprecia la incidencia de los mercados financieros globales sobre la rentabilidad de la Bolsa de Valores de Lima.

De los resultados expuestos en la tabla 5 , en el periodo evaluado, se aprecia que el mercado peruano tiene una relación directa importante con el mercado de Brasil con $r=0,943$, con el mercado de China con $r=0,687$, con el mercado de España con $\mathrm{r}=0,55$ y con Estados Unidos con $\mathrm{r}=0,438$. A partir de estos resultados se puede afirmar que los mercados financieros globales son interdependientes.

De acuerdo a los resultados y las demostraciones realizadas en el estudio, se puede afirmar, en concordancia con Perez(2010) que el comportamiento de la volatilidad de la Bolsa de Valores de Lima es heterocedástica, estacionaria, condicionada y persistente en el tiempo, lo que se ha demostrado con la prueba de Dickey-Fuller (tabl 1) y el Modelo $\operatorname{Garch}(1,1)$, teniendo en cuenta el valor ADF que es significativamente mayor que los valores críticos de Mackinnon a los 3 niveles de significancia $5 \%, 10 \%$ y $15 \%$.

Tabla 5.

Matriz de correlación de las principales bolsas del mundo

Fuente: Cotización de los mercados de valores, elaboración propia

\begin{tabular}{|c|c|c|c|c|c|c|}
\hline & & BVLIMA & IBEX35 & BOVESPA & SP500 & SHANGAI \\
\hline \multirow[t]{3}{*}{ BVLIMA } & Correlación de Pearson & 1 & $0,550^{\star \star}$ & $0,943^{\star \star}$ & $0,438^{\star \star}$ & $0,687^{\star \star}$ \\
\hline & Sig. (bilateral) & & 0,000 & 0,000 & 0,000 & 0,000 \\
\hline & $\mathrm{N}$ & 4283 & 4283 & 4283 & 4283 & 4283 \\
\hline \multirow[t]{3}{*}{ IBEX35 } & Correlación de Pearson & $0,550^{\star \star}$ & 1 & $0,597^{\star \star}$ & $0,839^{\star \star}$ & $0,627^{\star \star}$ \\
\hline & Sig. (bilateral) & 0,000 & & 0,000 & 0,000 & 0,000 \\
\hline & $\mathrm{N}$ & 4283 & 4283 & 4283 & 4283 & 4283 \\
\hline \multirow[t]{3}{*}{ BOVESPA } & Correlación de Pearson & $0,943^{\star \star}$ & $0,597^{\star \star}$ & 1 & $0,429^{\star \star}$ & $0,665^{\star \star}$ \\
\hline & Sig. (bilateral) & 0,000 & 0,000 & & 0,000 & 0,000 \\
\hline & $\mathrm{N}$ & 4283 & 4283 & 4283 & 4283 & 4283 \\
\hline \multirow[t]{3}{*}{ SP500 } & Correlación de Pearson & $0,438^{\star \star}$ & $0,839^{\star \star}$ & $0,429^{\star \star}$ & 1 & $0,477^{\star \star}$ \\
\hline & Sig. (bilateral) & 0,000 & 0,000 & 0,000 & & 0,000 \\
\hline & $\mathrm{N}$ & 4283 & 4283 & 4283 & 4283 & 4283 \\
\hline \multirow[t]{3}{*}{ SHANGAI } & Correlación de Pearson & $0,687^{\star \star}$ & $0,627^{\star \star}$ & $0,665^{\star \star}$ & $0,477^{\star \star}$ & 1 \\
\hline & Sig. (bilateral) & 0,000 & 0,000 & 0,000 & 0,000 & \\
\hline & $\mathrm{N}$ & 4283 & 4283 & 4283 & 4283 & 4283 \\
\hline
\end{tabular}

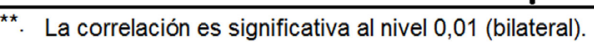




\section{REFERENCIAS BIBLIOGRÁFICAS}

Court, E. (2011). Estadísticas y Econometría Financiera. Lima: Editorial Pontificia Universidad Católica del Perú.

Enders, W. (2015). Applied Econometric Time Series. Alabama: University of Alabama.

Engle. R. (1982). Autoregressive Condicional Heteroscedasti city with Estimates of the variance of United Kingdom Inflation. Journal Econométrica, 50(4), pp. 987 - 1008.
Engle, R. (2002). Dynamic Conditional Correlation a simple class of Multivariate Garch Models. Journal of Business and Economic Statistics, 20(3), pp. 339-350.

Engle, R. (2003). Risk and Volatility Econometric Models and Financial Practice. The American Economic Review, 94(3), pp. 405-420.

Hernández, S. (2009). Pronóstico y Volatilidad del IPyC de la Bolsa Mexicana de Valores. Revista de la Universidad Cristóbal Colón, pp. 25-36. Recuperado de http://repec.ver.ucc.mx/ documents/num_1_ago_2009_004. pdf
Larios, J., Alvarez, V. y Quineche, R. (2014). Fundamentos de Econometría. Lima: Fondo Editorial de la Universidad San Ignacio de Loyola

Montenegro, A. (2010). Análisis de Series de Tiempo. Bogotá: Pontificia Universidad Javeriana.

Peña, D. (2010). Análisis de Series Temporales. España: Alianza Editorial

Perez, C. (2010). Econometría de Series Temporales. México: Editorial Pearson. 